\title{
Calcium-activated chloride channel regulator 1 as a prognostic biomarker in pancreatic ductal adenocarcinoma
}

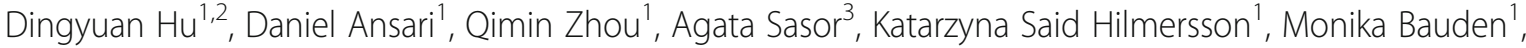 \\ Yi Jiang ${ }^{2}$ and Roland Andersson ${ }^{1 *}$ (i)
}

\begin{abstract}
Background: In a previous study utilizing mass spectrometry-based proteomics, we identified calcium-activated chloride channel regulator 1 (CLCA1) as a potential tumor suppressor in pancreatic cancer and the expression was inversely correlated with patient survival. The aim of the study was to further validate the prognostic significance of CLCA1 in pancreatic cancer.
\end{abstract}

Methods: CLCA1 expression was evaluated with tissue microarrays and immunohistochemistry in 140 patients with pancreatic ductal adenocarcinoma that underwent surgical resection at Skåne University Hospital, Sweden. KaplanMeier and Cox proportional hazards modeling were used to explore the association between CLCA1 and clinicopathological factors and survival.

Results: CLCA1 expression was denoted as positive in 90 tumors (64.3\%), with positive staining being limited to the tumor cells. There were no significant association between CLCA1 expression and established clinicopathological parameters. Low CLCA1 expression correlated significantly with shorter disease-free survival (11.9 vs 17.5 months, $P$ $=0.042$ ). Multivariable Cox regression analysis confirmed the results (HR 0.61, 95\% Cl-0.40-0.92, $P=0.019)$.

Conclusions: Low CLCA1 expression is an independent factor of poor disease-free survival in pancreatic cancer.

Keywords: Pancreatic ductal adenocarcinoma, CLCA1, Calcium-activated chloride channel regulators, Survival

\section{Background}

Pancreatic ductal adenocarcinoma (PDAC) is currently the third leading cause of cancer-related mortality [1]. Although achievements have been made to improve the diagnosis and treatment of PDAC, the five-year survival rate remains as low as 6\% [2]. Due to the silent progression of the disease and lack of early screening techniques, most patients are diagnosed at an advanced stage, precluding potentially curative surgery. Moreover, tumor heterogeneity is strongly implicated in the biological behavior of PDAC, as well as the response to therapy [3]. More information is needed concerning molecular factors that can contribute to an earlier diagnosis

\footnotetext{
* Correspondence: roland.andersson@med.lu.se

'Department of Surgery, Clinical Sciences Lund, Lund University and Skåne University Hospital, SE-221 85 Lund, Sweden

Full list of author information is available at the end of the article
}

and a better prediction of prognosis and treatment response.

Calcium-activated chloride channel regulators (CLCAs), also called "chloride channel accessory proteins", are a family of secreted self-cleaving proteins which activate calcium-dependent chloride currents. The human genome encodes 3 functional CLCA proteins, including CLCA1, CLCA2, and CLCA4. As one form of ion channels, $\mathrm{Ca}^{2+}$-activated chloride channels have been implicated in regulation of cell proliferation, cell migration and metastasis and are believed to be emerging therapeutic targets in cancer [4-6]. CLCA1 is mainly expressed in the large and small intestine and appendix, especially in crypt cells, and can be shed into the blood stream. It has been reported that CLCA1 can regulate the differentiation of colorectal cancer cells and function as a prognostic marker in colorectal cancer [7].

(c) The Author(s). 2018 Open Access This article is distributed under the terms of the Creative Commons Attribution 4.0 International License (http://creativecommons.org/licenses/by/4.0/), which permits unrestricted use, distribution, and reproduction in any medium, provided you give appropriate credit to the original author(s) and the source, provide a link to the Creative Commons license, and indicate if changes were made. The Creative Commons Public Domain Dedication waiver (http://creativecommons.org/publicdomain/zero/1.0/) applies to the data made available in this article, unless otherwise stated. 
Recent studies also supported that CLCA2 and CLCA4 may serve as tumor suppressors in breast cancer [8].

Our previous mass spectrometry-based proteomics study showed for the first time that CLCA1 is a biomarker for PDAC, with protein expression being 20 fold down-regulated in poor outcome PDAC patients [9]. The aim of the present study was to investigate the prognostic impact of CLCA1 in a large and well annotated clinical cohort of resectable PDAC patients.

\section{Methods}

\section{Patients and samples}

The REMARK guidelines were followed where possible throughout the whole study [10]. Formalin-fixed paraffin-embedded tissue samples were collected from 140 patients with PDAC who underwent pancreatic resection at the Department of Surgery, Skåne University Hospital, Lund and Malmö, Sweden, between 1996 and 2017. All tissue specimens were re-evaluated by a senior pancreas pathologist (A.S.) to ensure correct diagnosis and histopathological characterization. Disease-free survival (DFS) was defined as the time from pancreatectomy to the first evidence of clinical recurrence (locoregional or distant) or death from any cause. Overall survival (OS) was defined as the time from pancreatectomy to death from any cause or the last date the patient was seen alive. Ethical approval was obtained from the local human ethics committee at Lund University (Ref 2017/320).

\section{Tissue microarray}

Tumors with sufficient amount of material were deemed suitable for tissue microarray (TMA) construction. Compared with usage of whole sections, TMA has the advantage of a reduced consumption of both tissue and time which enables studies of a larger scale with reduced experimental variability [11]. Using an automated tissue arraying device (Minicore ${ }^{\bullet}$ 3, Alphelys, Plaisir, France), 4 cores $2 \mathrm{~mm}$ of cancer tissues (marked by pathologist A.S) from each specimen were stabilized into paraffin blocks. After a fine quality was assured, the TMA-blocks were sectioned for immunohistochemical analysis.

\section{Immunohistochemistry}

TMA-sections ( $3 \mu \mathrm{m}$ thick) were heated in $60{ }^{\circ} \mathrm{C}$ for $1 \mathrm{~h}$ and then cooled in room temperature (RT). Next, using automated PT Link (Dako, Glostrup, Denmark), deparaffinization, rehydration and antigen-retrieval were performed in EnVision FLEX Target Retrieval Solution high $\mathrm{pH}$ (K800421-2, Dako) heated to $96^{\circ} \mathrm{C}$ for $20 \mathrm{~min}$. After three times of wash in phosphate-buffered saline for $5 \mathrm{~min}$, sections were blocked against endogenous peroxidase activity with $0.3 \% \quad \mathrm{H}_{2} \mathrm{O}_{2}$ and $1 \%$ methanol in phosphate-buffered saline for $10 \mathrm{~min}$. The specimens were then blocked with $5 \%$ goat serum for $1 \mathrm{~h}$ at RT to reduce non-specific background staining, followed by avidin/biotin blocking kit (SP-2001, Vector Laboratories, Burlingame, CA, USA) for $15 \mathrm{~min}$ at RT, which reduces endogenous avidin and biotin activity. Subsequently, the sections were incubated with rabbit recombinant monoclonal CLCA1 antibody (Abcam, Cambridge, UK; cat no ab180851; dilution 1:2000) at $4{ }^{\circ} \mathrm{C}$ overnight. Next, sections were incubated with biotinylated secondary goat anti-rabbit antibodies (BA-1000, dilution 1:200, Vector Laboratories) for $1 \mathrm{~h}$ at RT. Following incubation with avidin-biotin-peroxidase complex (Vectastain Elite ABC-HRP Kit, PK-6100, Vector Laboratories) for $30 \mathrm{~min}$ at RT, the sections were incubated with chromogen diaminobenzidine (SK-4100, Vector Laboratories) for $5 \mathrm{~min}$. After washing in deionized water for $5 \mathrm{~min}$, nuclei were counterstained with Mayer's hematoxylin (Histolab, Gothenburg, Sweden) for $30 \mathrm{~s}$, and washed in tap water for another $5 \mathrm{~min}$. Finally, the specimens were dehydrated in graded alcohols and mounted using Pertex (Histolab). Negative controls were produced by omitting the primary antibodies. Slides were scanned for evaluation using an Aperio scanscope scanner (Leica Biosystems, Wetzlar, Germany). Variability between individuals was reduced by limiting sample preparation and analysis of expression to one professional respectively.

\section{Scoring procedure}

The immunostaining of CLCA1 was assessed semi-quantitatively by an experienced pancreas pathologist (A.S.) blinded to the clinical outcome. Staining below $10 \%$ was denoted as negative $(0)$. When $>10 \%$ of tumor cells were stained, expression was considered positive and denoted as mild (1), moderate (2) or strong (3) depending on the intensity. Samples with negative staining (0) and mild staining (1) were categorized as low expression group, while those with moderate (2) and strong (3) were categorized as high expression group.

\section{Statistical analysis}

Comparisons of categorical data were performed using Chi-square test or Fisher's exact test. Continuous data were compared by the Mann Whitney U test. KaplanMeier analysis and log rank test were used to illustrate differences in DFS and OS according to CLCA1 expression. Cox regression proportional hazards models were used for estimation of hazard ratios (HRs) for recurrence and death according to CLCA1 expression. Any variable with a $P$-value less than 0.25 was selected as a candidate for the mulvariable Cox regression analysis. In the iterative process of variable selection using forward, backward and stepwise selection covariates were removed from the model if they were non-significant and not a confounder as described by Hosmer-Lemeshow, 
resulting in the main effect model [12]. A $P$-value less than 0.05 was considered statistically significant. All the statistics were performed using STATA MP 14.1.

\section{Results}

\section{Patient cohort}

Baseline characteristics of patients with PDAC are presented in Table 1 . The median age was 69 years (interquartile range $63-73$ years) and $73(52.1 \%)$ were female. The estimated median DFS was 13.2 months and the estimated median OS was 25.0 months, respectively. One hundred thirteen $(80.7 \%)$ of patients received adjuvant chemotherapy.

\section{CLCA1 expression in PDAC}

CLCA1 expression was considered positive in 90 (64.3\%) of the 140 tumors. The expression of CLCA1 was

Table 1 Clinicopathological characteristics of patients with pancreatic ductal adenocarcinoma stratified by CLCA1 expression

\begin{tabular}{|c|c|c|c|c|}
\hline Factors & $\begin{array}{l}\text { Low CLCA1 } \\
N=87\end{array}$ & $\begin{array}{l}\text { High CLCA1 } \\
N=53\end{array}$ & $P$ value & Missing \\
\hline Age, years & $69(62-75)$ & $68(64-72)$ & 0.258 & \\
\hline Female gender & $44(50.6)$ & $29(54.7)$ & 0.634 & \\
\hline T-stage & & & 0.649 & $0.7 \%$ \\
\hline$-\mathrm{T} 1$ & $12(13.8)$ & $7(13.2)$ & & \\
\hline$-\mathrm{T} 2$ & $58(66.7)$ & $35(66.0)$ & & \\
\hline$-\mathrm{T3}$ & $16(18.4)$ & $10(18.9)$ & & \\
\hline$-\mathrm{T} 4$ & 0 & $1(1.9)$ & & \\
\hline N-stage & & & 0.485 & $1.4 \%$ \\
\hline$-\mathrm{NO}$ & $21(24.1)$ & $12(22.6)$ & & \\
\hline$-N 1$ & $30(34.5)$ & $24(45.3)$ & & \\
\hline$-\mathrm{N} 2$ & $34(39.1)$ & $17(32.1)$ & & \\
\hline $\begin{array}{l}\text { AJCC stage, } \\
\text { 8th edition }\end{array}$ & & & 0.835 & $1.4 \%$ \\
\hline$-I A$ & $4(4.6)$ & $2(3.8)$ & & \\
\hline$-\mid \mathrm{B}$ & $12(13.8)$ & $7(13.2)$ & & \\
\hline$-\| A$ & $4(4.6)$ & $3(5.7)$ & & \\
\hline$-\| \mathrm{B}$ & 31 (35.6) & $23(43.4)$ & & \\
\hline$-\mid I I$ & $34(39.1)$ & $18(34.0)$ & & \\
\hline Tumor differentiation & & & 0.879 & $1.4 \%$ \\
\hline - Well & $4(4.6)$ & $3(5.7)$ & & \\
\hline - Moderate & $28(32.2)$ & $20(37.7)$ & & \\
\hline - Poor & $50(57.5)$ & $29(54.7)$ & & \\
\hline - Undifferentiated & $3(3.4)$ & $1(1.9)$ & & \\
\hline R1 resection margin & $35(40.2)$ & $20(37.7)$ & 0.552 & $0.7 \%$ \\
\hline $\begin{array}{l}\text { Adjuvant } \\
\text { chemotherapy }\end{array}$ & $68(78.2)$ & $45(84.9)$ & 0.129 & $3.6 \%$ \\
\hline
\end{tabular}

Qualitative data are expressed as N (\%) and quantitative data as median (interquartile range). AJCC American joint committee on cancer limited to the tumor cells. Mild, moderate and strong staining of CLCA1 were present in 37 (26.4\%), 41 (29.3\%) and $12(8.6 \%)$ cases respectively. Figure 1 shows representative immunohistochemical images of CLCA1 expression in PDAC.

\section{Association between CLCA1 expression and clinicopathological characteristics}

The expression of CLCA1 was not associated with any traditional clinical parameters, including age, gender, TNM stage, histological grade, resection margin status and adjuvant chemotherapy.

\section{Association between CLCA1 expression and survival}

Kaplan-Meier analysis revealed that CLCA1 expression correlated with a significantly shorter DFS, with the worst outcome for tumors with low CLCA1 expression (Fig. 2). Median DFS was 11.9 months in patients with low CLCA1 expression and 17.5 months in patients with high CLCA1 expression, $P=0.042$. These findings were confirmed in univariable Cox regression analysis (HR 0.66, 95\% CI-0.44-0.99, $P=0.044$ ), and remained significant in multivariable analysis (HR 0.61, 95\% CI-0.40-0.92, $P=0.019$ ), adjusted for differentiation grade and resection margin status (Table 2). The OS was also reduced in patients with low CLCA1, but the association did not reach statistical significance. The median OS was 23.5 months in patients with low CLCA1 expression and 27.8 months in patients with high CLCA1 expression $(P>0.05)$ (Fig. 3).

\section{Discussion}

This study demonstrated that low CLCA1 expression is an independent factor of shorter DFS. This is in line with our previous proteomics work utilizing mass spectrometry [9], validating our findings with an orthogonal technique in a larger cohort.

Ion channels, in general, and $\mathrm{Ca}^{2+}$-activated chloride channels in particular, are known to be involved in the regulation of cell proliferation, cell migration and metastasis and are considered emerging cancer drug targets $[5,6]$. Several studies have reported that the CLCA1 expression is down-regulated in colorectal cancer tissues compared with adjacent normal tissues [13-16], with low CLCA1 expression predicting worse outcomes [7, 17]. Knockdown of CLCA1 in Caco-2 cell lines have been shown to inhibit cell differentiation and promote cell proliferation [15]. Further in-vitro experiments suggested that CLCA1 may function as a tumor suppressor in colorectal cancer by inhibiting the Wnt/beta-catenin signaling pathway and epithelial-mesenchymal transition, while in-vivo overexpression of CLCA1 led to inhibition of proliferation and metastasis [14]. 

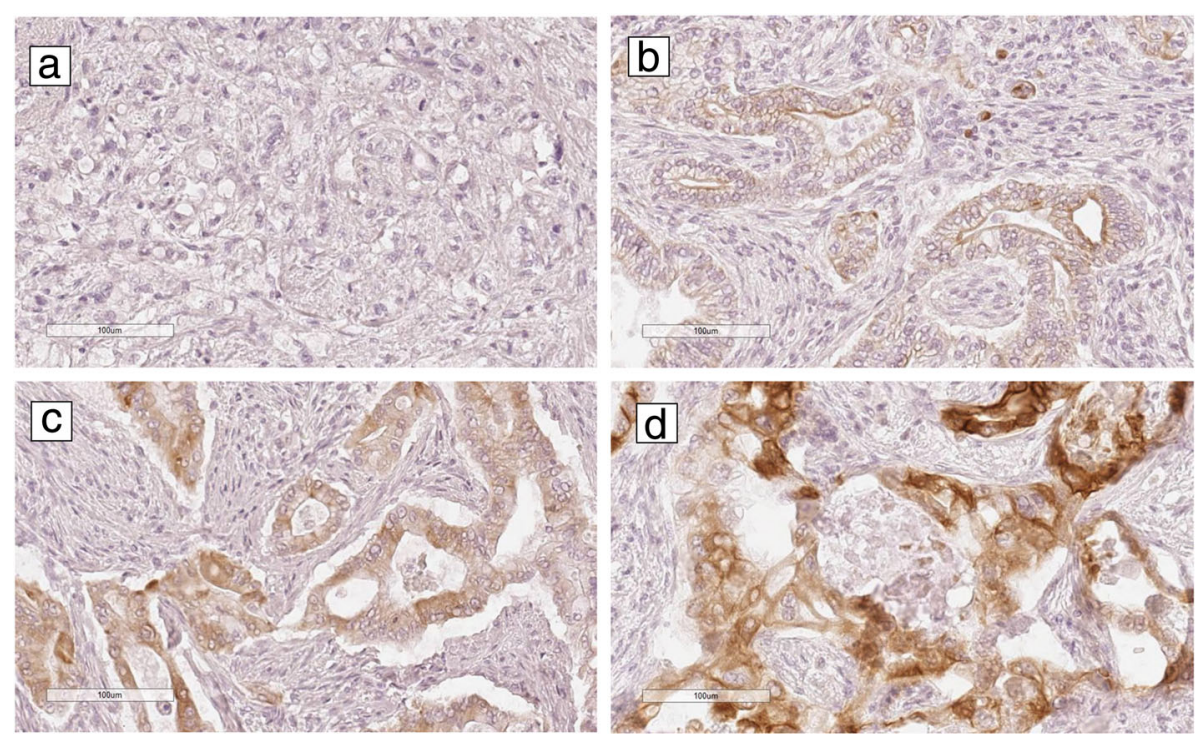

Fig. 1 Representative immunohistochemical images of CLCA1 expression in pancreatic ductal adenocarcinoma. a negative, b weak, c moderate, d strong staining

However, there is scant literature on the expression pattern and underlying function of CLCA1 in PDAC. Protein expression data in the Human Protein Atlas indicate that CLCA1 is mainly expressed in small and large intestines and appendix, while absent in both normal and cancerous pancreas tissues [18]. However, we noted that CLCA1 was present in more than half of pancreatic cancer tissues in our study. It is worth mentioning that CLCA1 can be secreted into pancreatic cyst fluid and the blood stream, which makes the CLCA1 a possible serum and fluid biomarker for PDAC [19]. Most recent evidence supported that CLCA1 mediates metalloprotease activity and is involved in intestinal mucus homeostasis by facilitating

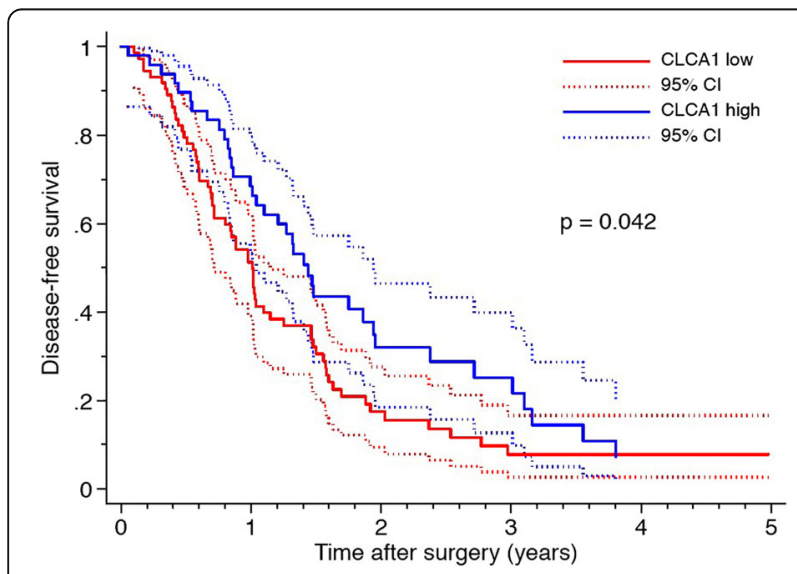

Fig. 2 Low expression of CLCA1 is associated with a poor DFS in pancreatic cancer patients undergoing surgical resection processing and removal of mucus [20]. This arises interest to address whether similar mechanisms are implicated in intraductal papillary mucinous neoplasm, a precursor of PDAC manifested with mucus contained cyst. Indeed, CLCA1 has been proposed as a supportive marker for high-grade dysplasia and malignant transformation using cyst fluid samples [19].

PDAC is characterized by a dense and heterogenous tumor microenvironment (TME), which drives tumor progression and resistance to therapy. While the past four decades have seen no decline in death rates of this devastating malignancy [1], a better understanding of the mechanisms how pancreatic cancer cell interactions with their TME might open new avenues of research in effective treatments of PDAC. Ion channels are involved in intracellular signaling events and activate specific cellular responses, including cancer-related proliferation, apoptosis, migration and angiogenesis [21]. Ion channels and their interactions with integrin in TME can contribute to tumor development and emerging drug targets [22]. For example, neutrophils in the TME release $\mathrm{Cl}^{-}$to accomplish their antimicrobial activity [23]. Furthermore, activated vascular endothelial cells are required for angiogenesis, in which $\mathrm{Ca}^{2+}$ permeable channels and $\mathrm{Ca}^{2+}$-dependent signaling play crucial roles [21]. Abdel-Gany et al. also confirmed that CLCAs facilitated vascular arrest of cancer cells via interacting with $\beta_{4}$ integrin and promote metastatic growth [23].

Secreted CLCA1 has been demonstrated to be a direct modulator of another calcium-dependent chloride channel, TMEM16A [24, 25]. CLCA1 can stabilize TMEM16A on the cell surface and prevent its internalization, thus 
Table 2 Univariable and multivariable Cox survival analyses for DFS

\begin{tabular}{|c|c|c|c|c|c|c|}
\hline \multirow[t]{2}{*}{ Variables } & \multicolumn{3}{|c|}{ Univariable analysis } & \multicolumn{3}{|c|}{ Multivariable analysis } \\
\hline & $\mathrm{HR}$ & $95 \% \mathrm{Cl}$ & $P$ value & $\mathrm{HR}$ & $95 \% \mathrm{Cl}$ & $P$ value \\
\hline Age & 0.98 & $0.96-1.00$ & 0.076 & & & \\
\hline Female gender & 0.66 & $0.45-0.98$ & 0.039 & & & \\
\hline T-stage & 1.14 & $0.82-1.58$ & 0.441 & & & \\
\hline $\mathrm{N}$-stage & 1.14 & $0.89-1.45$ & 0.311 & & & \\
\hline Differentiation grade & 1.48 & $1.06-2.07$ & 0.023 & 1.55 & $1.09-2.18$ & 0.014 \\
\hline Resection margin (R1) & 1.55 & $1.02-2.33$ & 0.036 & 1.63 & $1.07-2.49$ & 0.023 \\
\hline Adjuvant chemotherapy & 1.57 & $0.86-2.89$ & 0.144 & & & \\
\hline CLCA1 expression, high vs low & 0.66 & $0.44-0.99$ & 0.044 & 0.61 & $0.40-0.92$ & 0.019 \\
\hline
\end{tabular}

activating chloride currents [24, 25]. While the role of CLCA1 in PDAC remain unclear, TMEM16A was found to be overexpressed in PDAC cells and promote the cell migration [26]. TMEM16A has also been proposed to contribute to tumor growth and invasion of lung cancer, prostate cancer and head and neck squamous cell carcinomas [27-29].

In this study, CLCA1 predicted DFS, but not OS. Although DFS and OS are partly related, there are differences. In DFS, any type of recurrence or spread is counted as an event, including isolated local recurrences. Patients with low CLCA1 expression seemed to have more early recurrences, i.e. occurring within 1 year of surgery. Multimodal treatment of recurrent pancreatic cancer has been found to prolong survival [30, 31]. However, after 5 years of median follow-up, the number at risk in the patient cohort was only 11 patients due to the high mortality rate. Therefore, our sample size might be underpowered to show a statistically meaningful result in terms of OS.

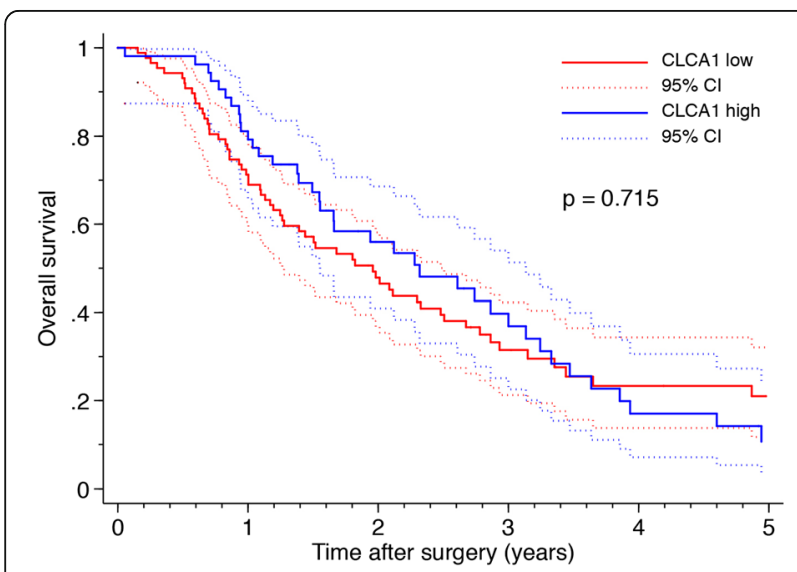

Fig. 3 Association between CLCA1 expression and OS in pancreatic cancer patients undergoing surgical resection

\section{Conclusion}

This study shows that low CLCA1 expression is a predictor of worse DFS in PDAC. CLCA1 may in the future be integrated into an immunohistochemistry panel to predict prognosis and treatment response in patients who undergo surgical resection. As ion channels have been suggested as emerging cancer drug targets, further investigation into the molecular mechanisms of CLCA1 in PDAC is needed.

\section{Abbreviations}

CLCA1: Calcium-activated chloride channel regulator 1; DFS: Disease-free survival; HRs: Hazard ratios; OS: Overall survival; PDAC: Pancreatic ductal adenocarcinoma; RT: Room temperature; TMA: Tissue microarray; TME: Tumor microenvironment

\section{Acknowledgements}

Not applicable.

The authors declare that they have no competing interests.

\section{Funding}

SWElife/Vinnova, the Magnus Bergvall Foundation, the Royal Physiographic Society of Lund, the Tore Nilsson Foundation, the Inga and John Hain Foundation for Medical Research, the Clas Groschinsky Foundation, the Gunnar Nilsson Foundation, the Crafoord Foundation and the Emil and Wera Cornell Foundation. The funding bodies played no role in study design, data collection or analysis, interpretation of data or manuscript writing.

\section{Availability of data and materials}

All relevant data and materials are included in the manuscript. For the full detailed data, please contact the corresponding author.

\section{Authors' contributions}

D.H. performed the experiments, analyzed the data and wrote the initial draft of the manuscript; D.A. collected the clinical data and follow-up of the patients, analyzed the data and assisted in manuscript writing and study conception; A.S. performed the histopathological evaluation of the tissue specimens; Q.Z., K.S.H., M.B., Y.J and R.A. critically revised the manuscript for important intellectual content; R.A. conceived the study; all authors read and approved the final manuscript.

\section{Ethics approval and consent to participate}

Ethical approval was obtained from the local human ethics committee at Lund University (Ref 2017/320). Written informed consent was given by participants in this study.

Consent for publication Not applicable. 


\section{Competing interests}

The authors declare that they have no competing interests.

\section{Publisher's Note}

Springer Nature remains neutral with regard to jurisdictional claims in published maps and institutional affiliations.

\section{Author details}

'Department of Surgery, Clinical Sciences Lund, Lund University and Skåne University Hospital, SE-221 85 Lund, Sweden. ${ }^{2}$ Department of Gastroenterology, The Second Affiliated Hospital and Yuying Children's Hospital of Wenzhou Medical University, Wenzhou 325017, China.

${ }^{3}$ Department of Pathology, Skåne University Hospital, 22185 Lund, Sweden.

Received: 30 August 2018 Accepted: 30 October 2018

\section{Published online: 12 November 2018}

\section{References}

1. Siegel RL, Miller KD, Jemal A. Cancer statistics, 2018. CA Cancer J Clin. 2018; 68(1):7-30

2. Gillen S, Schuster T, Meyer Zum Buschenfelde C, Friess H, Kleeff J. Preoperative/neoadjuvant therapy in pancreatic cancer: a systematic review and meta-analysis of response and resection percentages. PLoS Med. 2010; 7(4):e1000267.

3. Bailey P, Chang DK, Nones K, Johns AL, Patch AM, Gingras MC, et al. Genomic analyses identify molecular subtypes of pancreatic cancer. Nature. 2016;531(7592):47-52

4. Yurtsever Z, Sala-Rabanal M, Randolph DT, Scheaffer SM, Roswit WT, Alevy YG, et al. Self-cleavage of human CLCA1 protein by a novel internal metalloprotease domain controls calcium-activated chloride channel activation. J Biol Chem. 2012;287(50):42138-49.

5. Lang F, Stournaras C. Ion channels in cancer: future perspectives and clinica potential. Philos Trans R Soc Lond Ser B Biol Sci. 2014;369(1638):20130108.

6. Stock C, Schwab A. Ion channels and transporters in metastasis. Biochim Biophys Acta. 2015;1848(10 Pt B):2638-46.

7. Yang B, Cao L, Liu J, Xu Y, Milne G, Chan W, et al. Low expression of chloride channel accessory 1 predicts a poor prognosis in colorectal cancer. Cancer. 2015;121(10):1570-80.

8. Walia V, Ding M, Kumar S, Nie D, Premkumar LS, Elble RC. hCLCA2 is a p53inducible inhibitor of breast Cancer cell proliferation. Cancer Res. 2009; 69(16):6624-32.

9. Hu D, Ansari D, Pawlowski K, Zhou Q, Sasor A, Welinder C, et al. Proteomic analyses identify prognostic biomarkers for pancreatic ductal adenocarcinoma. Oncotarget. 2018;9(11):9789-807.

10. McShane LM, Altman DG, Sauerbrei W, Taube SE, Gion M, Clark GM, et al. REporting recommendations for tumour MARKer prognostic studies (REMARK). Br J Cancer. 2005:93(4):387-91.

11. Hassan S, Ferrario C, Mamo A, Basik M. Tissue microarrays: emerging standard for biomarker validation. Curr Opin Biotechnol. 2008;19(1):19-25.

12. Hosmer D Jr, Lemeshow S, Sturdivant R. Model-building strategies and methods for logistic regression. In: Applied logistic regression. 3rd ed. Hoboken, NJ: John Wiley \& Sons, Inc; 2013.

13. Yu J, Li X, Zhong C, Li D, Zhai X, Hu W, et al. High-throughput proteomics integrated with gene microarray for discovery of colorectal cancer potential biomarkers. Oncotarget. 2016;7(46):75279-92.

14. Li X, Hu W, Zhou J, Huang Y, Peng J, Yuan Y, et al. CLCA1 suppresses colorectal cancer aggressiveness via inhibition of the Wnt/beta-catenin signaling pathway. Cell Commun Signal. 2017;15(1):38.

15. Yang B, Cao L, Liu B, McCaig CD, Pu J. The transition from proliferation to differentiation in colorectal cancer is regulated by the calcium activated chloride channel A1. PLoS One. 2013;8(4):e60861.

16. Bustin SA, Li SR, Dorudi S. Expression of the Ca2+-activated chloride channel genes CLCA1 and CLCA2 is downregulated in human colorectal cancer. DNA Cell Biol. 2001;20(6):331-8.

17. Van den Broeck A, Vankelecom H, Van Eijsden R, Govaere O, Topal B. Molecular markers associated with outcome and metastasis in human pancreatic cancer. J Exp Clin Cancer Res. 2012;31:68.

18. Uhlen M, Oksvold P, Fagerberg L, Lundberg E, Jonasson K, Forsberg M, et al Towards a knowledge-based human protein atlas. Nat Biotechnol. 2010; 28(12):1248-50.
19. Jabbar KS, Arike L, Verbeke CS, Sadik R, Hansson GC. Highly accurate identification of cystic precursor lesions of pancreatic Cancer through targeted mass spectrometry: a phase IIc diagnostic study. J Clin Oncol. 2018; 36(4):367-75.

20. Nystrom EEL, Birchenough GMH, van der Post S, Arike L, Gruber AD, Hansson GC, et al. Calcium-activated Chloride Channel regulator 1 (CLCA1) controls mucus expansion in Colon by proteolytic activity. EBioMedicine. 2018:33:134-43.

21. Prevarskaya N, Skryma R, Shuba Y. Ion channels and the hallmarks of cancer. Trends Mol Med. 2010;16(3):107-21

22. Arcangeli A, Crociani O, Bencini L. Interaction of tumour cells with their microenvironment: ion channels and cell adhesion molecules. A focus on pancreatic cancer. Philos Trans R Soc Lond Ser B Biol Sci. 2014;369(1638): 20130101.

23. Abdel-Ghany M, Cheng HC, Elble RC, Lin H, DiBiasio J, Pauli BU. The interacting binding domains of the beta(4) integrin and calcium-activated chloride channels (CLCAs) in metastasis. J Biol Chem. 2003;278(49):49406-16.

24. Sala-Rabanal M, Yurtsever Z, Nichols CG, Brett TJ. Secreted CLCA1 modulates TMEM16A to activate $\mathrm{Ca}(2+)$-dependent chloride currents in human cells. elife. 2015:4:e05875.

25. Sala-Rabanal M, Yurtsever Z, Berry KN, Nichols CG, Brett TJ. Modulation of TMEM16A channel activity by the von Willebrand factor type a (MWA) domain of the calcium-activated chloride channel regulator 1 (CLCA1). J Biol Chem. 2017:292(22):9164-74.

26. Sauter DRP, Novak I, Pedersen SF, Larsen EH, Hoffmann EK. ANO1 (TMEM16A) in pancreatic ductal adenocarcinoma (PDAC). Pflugers Arch. 2015;467(7):1495-508

27. Cha JY, Wee J, Jung J, Jang Y, Lee B, Hong GS, et al. Anoctamin 1 (TMEM16A) is essential for testosterone-induced prostate hyperplasia. Proc Natl Acad Sci U S A. 2015;112(31):9722-7.

28. Godse NR, Khan N, Yochum ZA, Gomez-Casal R, Kemp C, Shiwarski DJ, et al. TMEM16A/ANO1 inhibits apoptosis via downregulation of Bim expression. Clin Cancer Res. 2017;23(23):7324-32.

29. Jia L, Liu W, Guan L, Lu M, Wang K. Inhibition of calcium-activated Chloride Channel ANO1/TMEM16A suppresses tumor growth and invasion in human lung Cancer. PLoS One. 2015;10(8):e0136584.

30. Sperti C, Moletta L, Merigliano S. Multimodality treatment of recurrent pancreatic cancer: Mith or reality? World J Gastrointest Oncol. 2015;7(12):375-82.

31. Groot VP, van Santvoort HC, Rombouts SJ, Hagendoorn J, Borel Rinkes $\mid H$, van Vulpen $M$, et al. Systematic review on the treatment of isolated local recurrence of pancreatic cancer after surgery; re-resection, chemoradiotherapy and SBRT. HPB (Oxford). 2017;19(2):83-92.

\section{Ready to submit your research? Choose BMC and benefit from:}

- fast, convenient online submission

- thorough peer review by experienced researchers in your field

- rapid publication on acceptance

- support for research data, including large and complex data types

- gold Open Access which fosters wider collaboration and increased citations

- maximum visibility for your research: over $100 \mathrm{M}$ website views per year

At BMC, research is always in progress.

Learn more biomedcentral.com/submission 